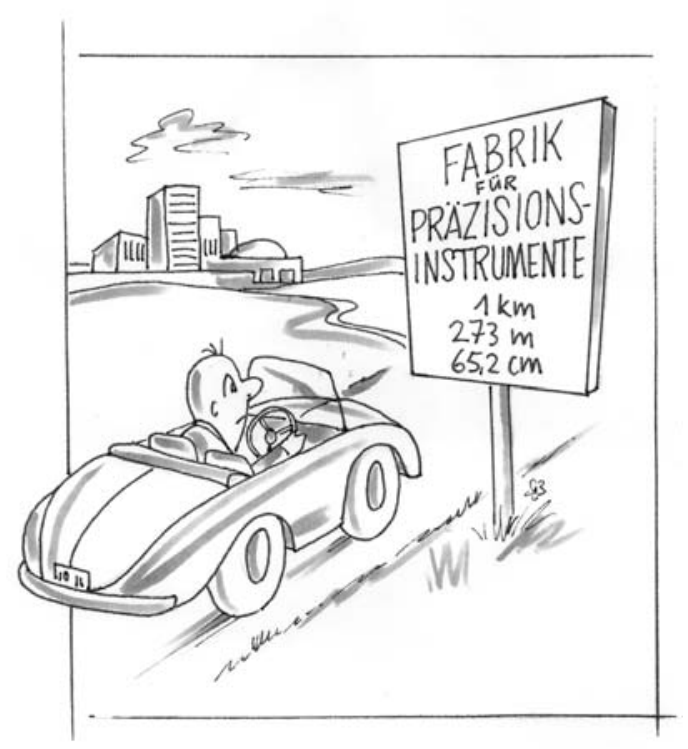

\title{
Ewig und drei Tage
} von Alfred Schreiber

Die folgende kleine Plauderei betrifft den bisweilen unerwarteten Nutzen falscher Genauigkeit. - So mancher der Mathematik Fernstehende mag denken: Je genauer, umso besser. Exaktheit ist die Zierde der Wissenschaft, Präzision das Gütesiegel der Technik. Aber ganz so einfach liegen die Dinge nicht. Obwohl Pünktlichkeit die Höflichkeit der Könige sein soll, verabredet man sich - außer vielleicht bei Banküberfällen - doch nicht auf die Sekunde genau (schon gar nicht bei einem Rendezvous), zu schweigen von der Frage, wie strikt die Partner sich tatsächlich an die vereinbarte Uhrzeit halten. Und welcher vernünftige Mensch würde sich je anheischig machen, einen Acker millimetergenau $\mathrm{zu}$ vermessen!

Dass so etwas ins Reich des Humors gehört, hat Walter Lietzmann in Lustiges und Merkwürdiges von Zahlen und Formen (9. Auflage, Göttingen 1961) mit zwei witzigen Kostproben belegt: „Im Museum erklärt der Führer: ,Diese Mumie ist 5007 Jahre alt. ,Woher wissen Sie das so genau?’ ,Ja, sehen Sie, als ich hier angestellt wurde, da war die Mumie 5000 Jahre alt - und das ist jetzt gerade 7 Jahre her." " Das zweite Beispiel parodiert das immer wieder einmal durch Wirklichkeitsferne bedrohte traditionelle Sachrechnen: „Auf dem Dach sitzen sieben Spatzen. Der Vater schießt und trifft zwei. Wie viele bleiben sitzen?"

Tatsächlich erweist sich auch realer Mathematikunterricht nicht selten als Hort von falscher Genauigkeit, was subjektiv erheitern würde, wäre es objektiv manchmal nicht doch etwas ärgerlich. Zum Beispiel berichtete vor einiger Zeit Wolfgang Kroll (in einem Leserbrief in den Mitteilungen 3-2003), Schüler sollten in Pflichtaufgaben hessischer Abschlussprüfungen die Höhe einer in ihren Maßen ägyptisch anmutenden Pyramide (136 m Basislänge) exakt zu 104,71 m berechnen - oder gar für eine Brauerei ermitteln, wie viele Glas Bier in eine hohle Litfasssäule passen, nämlich 45,91 Stück.

Nach Friedrich Hegel ziehen „die Worte: ewig, heilig, absolut, unendlich ... den Menschen, der etwas dabei fühlt, in die Höhe, erwärmen, erhitzen ihn“. Hegel war Philosoph. Dichtkunst hingegen, wenn sie plumpes Pathos vermeiden will, verfügt über subtilere Mittel, eine solche Aura zu evozieren. Eines dieser Mittel ist der metaphorische Gebrauch von falscher Genauigkeit. Der argentinische Schriftsteller Jorge Luis Borges hat darauf in einer 1967/68 in Harvard abhaltenen Vortragsreihe aufmerksam gemacht. Als Beispiel dient ihm eine Gedichtzeile eines von Kipling zitierten (von Borges nicht mehr erinnerten) Autors: „A rose-red city, half as old as time". Hierzu Borges:

Hätte der Dichter geschrieben: A rose-red city, as old as time, hätte er so gut wie gar nichts geschrieben. Aber ,halb so alt wie die Zeit' gibt allem eine Art magischer Genauigkeit ....

In ähnlicher Weise diente im 17. Jahrhundert gelegentlich die Zahl vierzig der Anspielung auf Nichtendendes oder Vieles, etwa in der von Shakespeare stammenden Zeile: „Wenn vierzig Winter deine Stirn belagern ..." (vgl. J. L. Borges: Das Handwerk des Dichters, Hanser: München 2002, S. 32). 
Die Zahl 1000 ist ein anderes Beispiel. Tausend mag für ,viel' stehen, aber tausendundeine Nacht mutet an wie eine kleine Ewigkeit. Auf bemerkenswerte Weise wird dabei die absichtsvolle Vagheit beim alltäglichen Gebrauch von Tausend konterkariert. Denn in der Tat hält sich das berühmte arabische Geschichtenbuch exakt an die im Titel vorgegebene Zahl, indem die Erzählerin jeweils bei Tagesanbruch eine Pause einlegt (aus gutem Grund immer an einer spannenden Stelle): „Da bemerkte Schehrezâd, daß der Morgen begann, und sie hielt in der verstatteten Rede an." (so die Übertragung Enno Littmanns). Die 1000-fache Unterbrechung und anschließende Wiederaufnahme des Erzählfadens mit immer derselben Überleitungsphrase lässt schließlich schiere Quantität in magisch anmutende Qualität umschlagen und erzeugt, zumindest im empfänglichen Leser, das von Hegel beschworene Gefühl der Erhebung, vorausgesetzt, man schafft es bis zur letzten Nacht, von der es heißt: „Jene Nacht zählte zum irdischen Leben nicht, und ihre Farbe war weißer als des Tages helles Angesicht."

Mein letztes Beispiel hat nichts Magisches an sich und macht auf den ersten Blick eher den Eindruck, als habe jemand falsch gezählt. Es handelt sich um das Gedicht Kinderpredigt, das sich im Kinderlieder-Anhang zu Des Knaben Wunderhorn findet (eine berühmte Sammlung von Volkspoesie, die Achim von Arnim

\section{Oberwolfach Lecture}

Am 16.10.2005 findet am Mathematischen Forschungsinstitut Oberwolfach (MFO) im Anschluss an die Mitgliederversammlung der Gesellschaft für mathematische Forschung e. V. die erste Oberwolfach Lecture statt.

Vortragender ist Prof. Dr. Gerhard Huisken, MaxPlanck-Institut für Gravitationsphysik (Golm). Der

\section{Arbeitsgemeinschaft im MFO}

Die nächste Arbeitsgemeinschaft im Mathematischen Forschungsinstitut Oberwolfach findet vom 2. bis 8 . Oktober 2005 zum Thema

Modern Foundations for Stable Homotopy Theory

unter der Leitung von John Rognes (Oslo) und Stefan Schwede (Bonn) statt. und Clemens Brentano Anfang des 19. Jahrhunderts herausgegeben haben). Der skurrile Text reflektiert eine Kanzelrede in sprunghaftem Duktus und kindersprachlicher Verballhornung von unverstandenen (oft lateinischen) Namen. Ich zitiere hier nur die letzte Strophe, mit der sich in surrealistischen oder DadaZirkeln gewiss Ehre hätte einlegen lassen:
Und die drei Schwestern Lazari,
Katharina, Sibilla, Schweigstilla,
Weinten bitterlich
Und der Hahn krähete Buttermilch!

Anders als hier behauptet, hat Lazarus (nach Joh. 11) nur zwei Schwestern mit Namen Maria und Martha. An die konnte oder wollte sich der Verseschmied offenbar nicht erinnern. Mehr noch fügt er ihnen, bei ausdrücklicher Nennung der neuen Anzahl, eine weitere Schwester hinzu, von der man bis dahin noch nichts gehört hatte. Ihr Name mag dies erklären.

\section{Adresse des Autors}

Prof. Dr. Alfred Schreiber

Institut für Mathematik und ihre Didaktik

Universität Flensburg

Auf dem Campus 1

24943 Flensburg

alfred.schreiber@uni-flensburg.de

einstündige Vortrag mit dem Titel „Geometrische Evolutionsgleichungen und die Uniformisierung Riemannscher Mannigfaltigkeiten" beginnt am MFO um 11:30 Uhr.

Die Veranstaltung ist öffentlich, Gäste sind herzlich willkommen. Wegen des beschränkten Platzes bitten wir um vorherige Anmeldung unter admin@mfo.de.
Das genaue Programm mit Hinweisen zur Teilnahme wird im Vorfeld auf der Homepage von Oberwolfach (www.mfo.de) veröffentlicht. Dort finden sich auch Informationen zu den weiteren Veranstaltungen des Instituts, insbesondere Hinweise für die Bewerbung zu den Oberwolfach-Seminaren im Oktober und November 2005 . 